Particle Accelerators, 1990, Vol. 28, pp. 195-200 Reprints available directly from the publisher Photocopying permitted by license only
(C) 1990 Gordon and Breach, Science Publishers, Inc. Printed in the United States of America

\title{
THREE-DIMENSIONAL COMPUTATION OF MAGNETIC FIELDS AND LORENTZ FORCES OF AN LHC DIPOLE MAGNET USING THE METHOD OF IMAGE CURRENTS
}

\section{DAUM}

NIKHEF-H, Amsterdam, Netherlands

D. TER AVEST

Applied Superconductivity Centre, University of Twente, Enschede, Netherlands

\begin{abstract}
Magnetic fields and Lorentz forces of an LHC dipole magnet are calculated using the method of image currents to represent the effect of the iron shield. The calculation is performed for coils of finite length using a parametrization for coil heads of constant perimeter. A comparison with calculations based on POISSON and TOSCA is made.
\end{abstract}

\section{INTRODUCTION}

For the design of magnets, a detailed knowledge of fields and forces is needed as well in the straight sections of the coil as in the coil heads. The method of computation presented here is designed for structures with shell coils around a cylindrical aperture surrounded by a cylindrical iron yoke. The effect of the iron is taken into account using the method of image currents for a fixed value of the permeability, and, hence, the variation of the permeability in the iron is not taken into account. The fields and forces are calculated as the sum of the fields and forces of the strands out of which the conductors are composed. The strands are "ideal" strands, i.e. they are parallel to the axis of the conductor and thus do not follow the actual layout of the strands in a Rutherford cable. The current is concentrated in the centre of the strands. The magnetic field due to a single strand is calculated with the Biot-Savart law using delta functions for the radial and angular current distributions. The integrals in the Biot-Savart law can now be evaluated. Fields are always calculated as the sum of the contributions of the individual strands. A detailed description ${ }^{1}$ is used for constant perimeter coil heads. Lorentz forces are calculated at the centre of either the strands, or the conductors, or the blocks with the current concentrated at the centre. Results on magnetic fields and field integrals, a multipole expansion of the field integrals, and the magnetic length are presented. 


\section{AN LHC DIPOLE MAGNET}

The shell coil of the prototype LHC magnet ${ }^{2,3}$ consists of two layers. A quadrant of the coil is shown in Fig. 1, which also defines the coordinate system used. The inner layer has four blocks of conductors with four, four, three and two conductors, the outer layer has two blocks of conductors with seven and seventeen conductors The conductors in the inner layer consist of two rows of thirteen "ideal" strands, those in the outer layer have two rows of twenty "ideal" strands. Both conductors have a slight keystone angle. Fig. 1 also shows a cross section of both conductors, which have a height of $17 \mathrm{~mm}$, and have a width at top and bottom of.2.64 (1.81)mm and 2.18(1.44)mm for layer $1(2)$, respectively.

The LHC prototype magnet is a twin aperture magnet. The method of computation described in this paper is for a single aperture magnet with a cylindrically symmetric configuration. The shape of the "ideal" strands in the conductors is a race track with straight parts with individual length for each strand and with coil heads, which are half circles, if exposed in a flat plane, at the inner radius of the conductor, and half ellipses elsewhere for obtaining constant perimeter coil ends. The coordinate system is defined with the $\mathrm{x}$-axis in the horizontal plane transverse to the symmetry axis of the magnet, the $y$-axis along the field direction and the z-axis along the symmetry axis.

a)

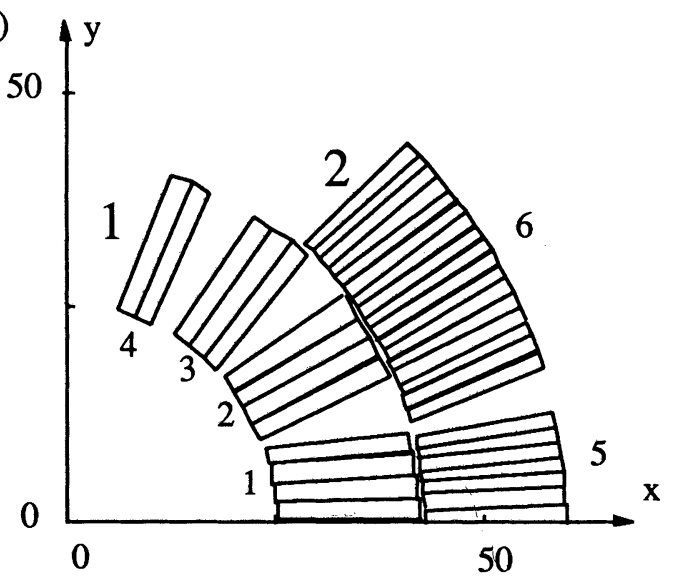

b)

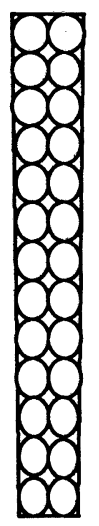

1

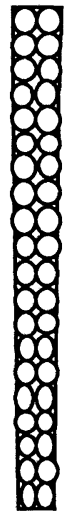

2

FIGURE 1 Layout a) of the conductors in the 6 current blocks of an LHC dipole magnet with dimensions in $\mathrm{mm}$, and b) of the "ideal" strands in the conductors of layer 1 and 2. 


\section{COMPUTATION OF FIELDS, FIELD INTEGRALS AND FORCES}

The basic integrals of the Biot-Savart law are expressed in a cylindrical coordinate system with the z-axis as symmetry axis of the cylinder. They can be evaluated analytically for the straight parts of each strand, if the current is taken to be concentrated at the centre of the "ideal" strands of Fig. 2. The integrals over the angular variables can be performed immediately using a delta function for the radial and azimuthal distributions at the position of the strand. The integration over $\mathrm{z}$ can be performed analytically for the exact length of the straight part of the strand. In the coil heads, the integration over the radial and angular variables can again be performed analytically. The integration over $\mathrm{z}$ is made using the Simpson rule, for which it is sufficient to make only 10 steps over a coil head. The contribution of all strands at any field point is obtained by summation over the contributions of all strands'. In the same way, also field integrals are calculated.

The iron yoke has a radius r' in the central part of the magnet and jumps to a radius $r$ '" in the outer part before the straight parts of the coils end. These radii are used for the calculation of the position of the image current of each strand and their contribution to the magnetic field as for the direct contributions of the straight parts of the strands. The following approximations are made. It is assumed that the iron yoke extends to infinity in the $z$-direction as well as in the radial direction outwards from radius r' and r' 'separately. Images with respect to the plane transverse to the z-axis where the actual iron yoke changes from inner radius r' to $r^{\prime \prime}$ are neglected. The relative permeability of the iron is taken to be $\mu=\infty$.

A comparison ${ }^{1}$ with three-dimensional field calculations using TOSCA $^{4}$ and with the multipoles of a two-dimensional calculation using POISSON's shows that these approximations can be made in the case that the radii r' and r" are sufficiently large, and hence the contribution of the image currents to the field is small.

The Lorentz force on a point of a strand is the vector product of the strand current and the field at this point due to all other strands. It has been checked' that the force on a conductor, taking the current to be concentrated at the centre of the conductor, is within a few percent equal to the sum of the forces on the strands of the conductor. The forces on the conductors are used in the mechanical design of the magnet. In all cases, the field and field integrals are calculated from the individual strands. 


\section{RESULTS FOR AN LHC MAGNET}

A program has been written for field and force computations with the method presented above. It has been applied to an LHC magnet of a nominal length of $1 \mathrm{~m}$ with the conductor layout of Fig.1. The field and force configuration has been calculated for a central field $\mathrm{B}(0,0,0)=10 \mathrm{~T}$ for which an excitation current $\mathrm{I}=14375 \mathrm{~A}$ is needed. Then, the current in the strands of layer 1 and layer 2 are $I_{1 s}=552.9 A$ and $I_{2 s}=359.4 \mathrm{~A}$, respectively. The corresponding current densities are about $\mathrm{J}_{1 \mathrm{~s}}=404 \mathrm{~A} / \mathrm{mm}^{2}$ and $\mathrm{J}_{2 \mathrm{~s}}=558 \mathrm{~A} / \mathrm{mm}^{2}$, respectively. A graded current density is used for optimization of the current carrying capability of available conductors for the two coil layers. The iron yoke has an inner radius $r^{\prime}=0.100 \mathrm{~m}$ between $\mathrm{z}=-0.302 \mathrm{~m}$ and $\mathrm{z}=0.302 \mathrm{~m}$. Outside this range, it has an inner radius $\mathrm{r} "=0.128 \mathrm{~m}$. The contribution of the image currents in the iron yoke is less than $15 \%$ due to the large inner radius of the iron.

An important design criterion is the maximum field on the conductors. This occurs in layer 1 at the inner top edge of block 4 , and in layer 2 close to the inner top edge of block 6 (see Fig. 1). The maximum fields in layer 1 and layer 2 are $B_{1_{\max }}=10.217 \mathrm{~T}$, and $\mathrm{B}_{2 \max }=$ $8.772 \mathrm{~T}$ at $\mathrm{z}_{1}=0 \mathrm{~m}$, and $\mathrm{z}_{2}=0.36 \mathrm{~m}$, respectively. In layer 2 the field at $\mathrm{z}=0$ equals $8.591 \mathrm{~T}$. These maxima should be compared with the properties of available superconductors at the required current densities. The magnetic length of this configuration is $\mathrm{L}_{\text {magn }}=0.867 \mathrm{~m}$.

For a further detailed understanding, Fig. 2 shows the direct contributions of the coils to the field on the $\mathrm{z}$-axis and to the usual multipole coefficients $\mathrm{c}_{\mathrm{n}}$ of the field integrals, and those of their mirror images in the iron yoke for a magnet of $1 \mathrm{~m}$ nominal length. We see that all multipole coefficients due to the mirror images for $\mathrm{n}=5$ and larger are negligibly small.
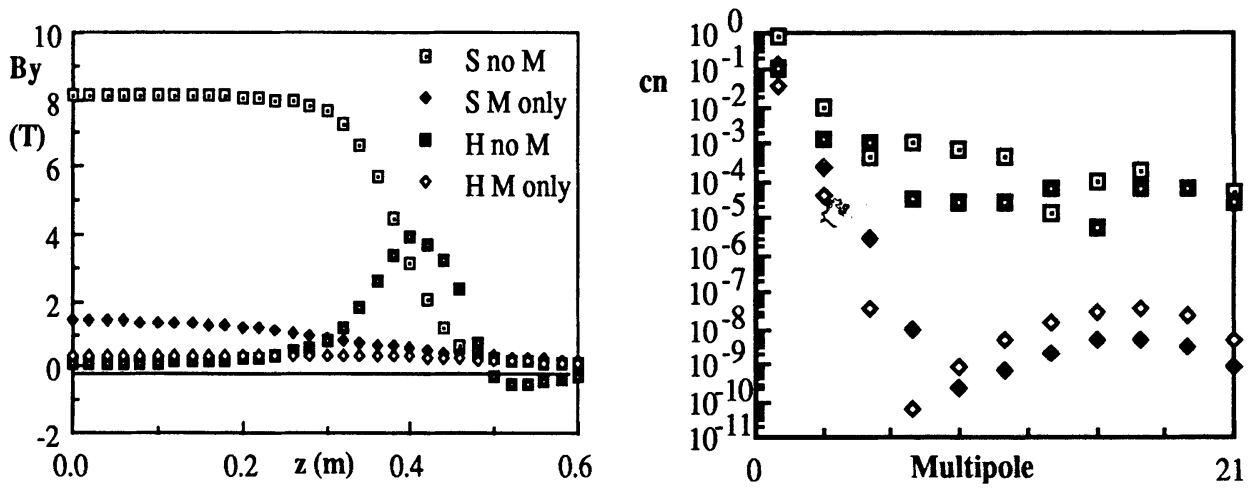

FIGURE 2 Field along the $\mathrm{z}$-axis and multipole coefficients for direct contribution of the straight parts of the strands ( $\mathrm{S}$ no $\mathrm{M})$ and the coil heads $(\mathrm{H}$ no $\mathrm{M})$, and for their mirror contribution (S M only and H M only). 

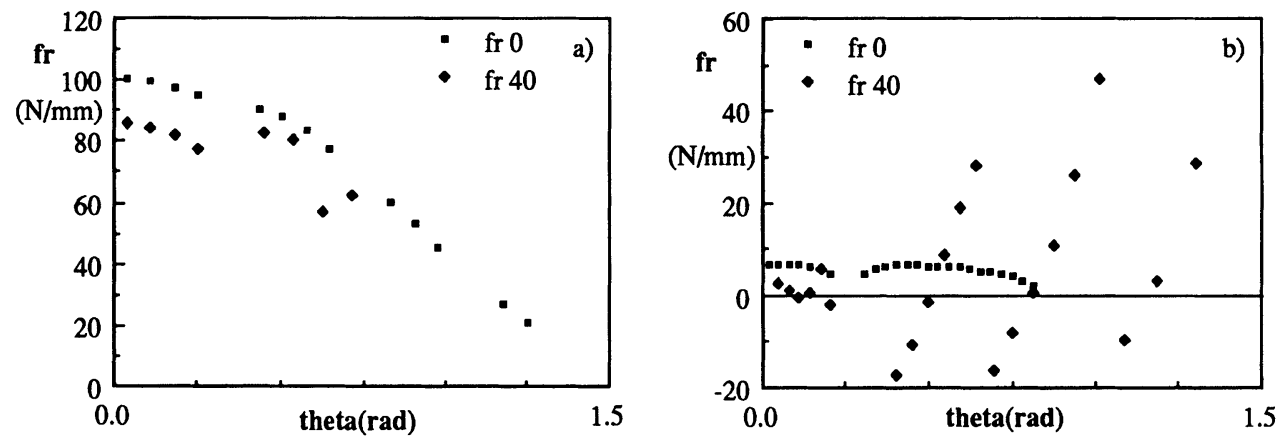

FIGURE 3 Radial component of Lorentz force at $\mathrm{z}=0$ and $40 \mathrm{~cm}$ in a) layer 1 , b) layer 2 .

Fig. 3 shows the radial component of the Lorentz force as function of the azimuthal angle $\theta$ of the conductors in the straight part of the coils at $z=0$ and $40 \mathrm{~cm}$, respectively. The radial force is always outward and at $\mathrm{z}=0 \mathrm{~cm}$. The reversal of radial forces at $\mathrm{z}=40 \mathrm{~cm}$ within blocks needs careful attention in the mechanical design of a magnet. The overall radial force of the two layers is outward, which implies, however, a compression of the insulation between inner and outer layer. The azimuthal force is always towards the median plane. Hence, all forces are contained within the arch of the coil, and no forces are exerted on the beam pipe. The accumulated azimuthal force towards the median plane adds up to a very high stress on the conductors in the median plane. The azimuthal stress for layer 1 and layer 2 are about $\sigma_{1 \mathrm{a}}=57 \mathrm{~N} / \mathrm{mm}^{2}$, and $\sigma_{2 \mathrm{a}}=70 \mathrm{~N} / \mathrm{mm}^{2}$, respectively. The azimuthal forces require a high azimuthal prestress, which add to the stress values, and therefore, can not be a comfortable factor of more than two higher than the magnetic force at maximum excitation as in the case of the HERA magnets ${ }^{6}$. Also the radial force on e.g. the first conductor of block 1 causes a very high radial stress of about $\sigma_{1 \mathrm{r}}=34 \mathrm{~N} / \mathrm{mm}^{2}$. These values require strong attention in view of the properties of the copper matrix of superconducting cables, in particular for $\mathrm{Nb}_{3} \mathrm{Sn}$ conductors. The total longitudinal forces for one quadrant in the first layer and the second layer are outward and amount to $f_{1 z}=27.6 \mathrm{kN}$, and $f_{2 z}=70.1 \mathrm{kN}$, respectively, requiring strong end plates.

For the HERA dipole magnets ${ }^{6}$ a study was made of the influence of the spacing between conductors in the coil heads on the maximum field in the conductor locally. The contribution of the sextupole component to the field integral was minimized locally at the coil head. For the LHC magnet, the sextupole contributions of the straight part of the strands and those of the coil heads have opposite sign for the configuration of Ref. 3 and a closed configuration of the blocks, as shown in Table I. This permits the design of a coil head with a minimal sextupole component. 
TABLE I Comparison of the sextupole component of the field integral for the configuration of Ref. 3 and a closed configuration.

$\begin{array}{rrrrr}\text { Length } & \text { Configuration } & b_{3 \text { total }} & b_{3 \text { straight }} & b_{3 \text { head }} \\ 1 \mathrm{~m} & \text { open } & 9.17 * 10^{-3} & 1.07 * 10^{-2} & -1.50 * 10^{-3} \\ 1 \mathrm{~m} & \text { closed } & -4.11 * 10^{-4} & 1.09 * 10^{-3} & -1.50 * 10^{-3}\end{array}$

Fig. 4 shows the comparison ${ }^{1}$ between the multipoles for a $1000 \mathrm{~m}$ magnet using our method and a two-dimensional POISSON calculation for the configuration of Fig. 1. Taking the same current for both cases, the direct contributions of the strands to $\mathrm{B}(0,0,0)=10 \mathrm{~T}$ are the same. Scaling of the mirror contribution results in an average relative permeability $\mu=$ 16.3 for our calculation. A similar comparison ${ }^{1}$ with a three-dimensional TOSCA calculation yields $\mu=18.4$.

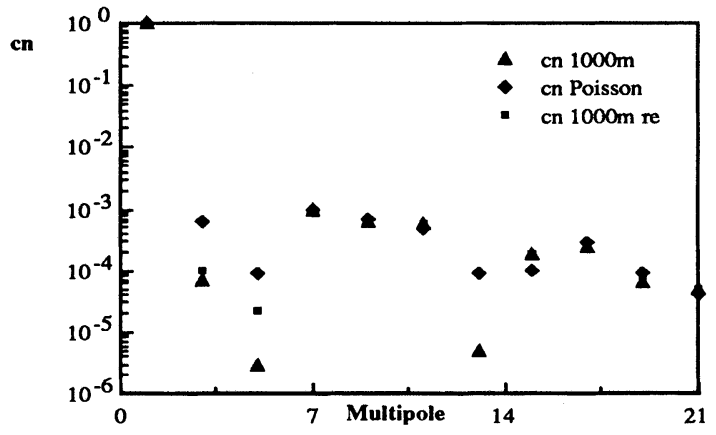

FIGURE 4 Multipoles for a 1000m long magnet, a two-dimensional POISSON ${ }^{5}$ calculation, and a rescaled $1000 \mathrm{~m}$ magnet with same average relative $\mu$ as in the POISSON calculation.

\section{REFERENCES}

1. C. Daum and D. ter Avest, Three-dimensional computation of the magnetic fields and Lorentz forces of an LHC dipole magnet, NIKHEF-H 89/12 and LHC note No. 94.

2. The large Hadron Collider in the LEP Tunnel, ed. by G. Brianti and K. Hübner, CERN 87-05, 27 May 1987.

3. D. Leroy, R.Perin, G. de Rijk, W. Thomi, Design of a High Field Twin Aperture Superconducting Dipole Model, CERN SPS/87-32 (EMA), LHC note No 62.

4. TOSCA, Vector Fields, Oxford, UK.

5. POISSON, CERN program library, T604.

6. K.H. Mess, P. Schmüser, Superconducting Accelerator Magnets, DESY HERA 8901, and in the Proceedings of the CERN Accelerator School on Superconductivity in Particle Accelerators, Ed. S. Turner, CERN 89-04, p.87. 